\title{
A New Nonuniform Frequency Sampling Technique for Multidimensional FIR Filter Design
}

\author{
Avideh Zakhor and Gary Alvstad \\ Department of Electrical Engineering and Computer Sciences \\ University of California \\ Berkeley, CA 94720 \\ (415) 643-6777
}

Multidimensional (M-D) digital filtering is of extreme importance in many signal processing applications including image, video, geophysical, and biomedical data processing. Some of the existing techniques for M-D Finite Impulse Response (FIR) filter design include window, transformation, and frequency sampling methods. In this paper, we derive a new nonuniform frequency sampling method for designing M-D FIR filters. The major advantage of this technique over window and transformation methods is its simplicity, and its advantage over existing frequency sampling methods is its increased flexibility.

Uniform frequency sampling techniques for design of M-D FIR filters involve taking the inverse discrete Fourier transform of samples of a desired frequency response at the vertices of a uniform 2-D Cartesian grid. Although this technique is computationally efficient, it is not flexible in locating frequency samples. A major difficulty with any nonuniform frequency sampling technique results from the fact that, unlike the one-dimensional (1-D) case, samples of an M-D polynomial at arbitrary locations may not result in unique recovery of its coefficients. However, recent results in multivariate polynomial interpolation theory indicate that mild restrictions on the locations of the samples of a polynomial result in its unique reconstruction [1]. Specifically, an M-D polynomial has been shown to be uniquely reconstructible from its samples on irreducible curves, provided there are enough samples on each curve. Our approach is to exploit this result in order to derive a nonuniform frequency sampling technique for the M-D filter design problem. This technique entails choosing an appropriate number of samples of the desired frequency response on an appropriate number of straight lines with different rational slopes in such a way that the resulting filter design problem has a unique solution.

A problem distinct from that of uniquely specifying filters with their nonuniform frequency samples is that of developing efficient algorithms for determining their coefficients. We propose three strategies for doing this. The first one is a recursive approach in which slopes of the sampling lines are chosen to be fixed integers. The second one is a line by line reconstruction approach in which frequency samples are first used to determine coefficients of 1-D filters associated with the sampling lines. These coefficients are subsequently utilized to specify coefficients of the two-dimensional filter. The third approach involves solving a linear system of equations, which is guaranteed to have a unique solution provided the nonuniform frequency samples satisfy the theoretical constraints.

Filter design examples associated with the above three approaches will be presented. We will also discuss design issues related to optimal choice of location and slopes of the sampling lines, and distribution of the sampling points within them. Finally, performance characteristics of the proposed design methodology is compared to that of existing M-D filter design techniques. 


\section{References}

1. A. Zakhor and A. V. Oppenheim, "Sampling Schemes for Reconstruction of Multidimensional Signals from Multiple Level Threshold Crossings," Proc. Int. Conf. on Acoustics, Speech and Signal Processing, New York, April 1988. 